\title{
Temperatura base, coeficientes de cultura e graus-dia para cafeeiro arábica em fase de implantação ${ }^{1}$
}

\author{
Edson P. Lima ${ }^{2} \&$ Elio L. da Silva ${ }^{3}$
}

\begin{abstract}
RESUMO
Este trabalho teve como propósito determinar as temperaturas base inferior - Tb, e superior - TB, para cafeeiro arábica. Avaliaram-se, portanto, as cultivares Acaiá Cerrado (MG-1474) e Rubi (MG-1192), com as quais se promoveu o estudo associando-se coeficientes de cultura - Kc, a graus-dia de desenvolvimento - GDD, na Universidade Federal de Lavras - UFLA, entre março de 2003 e setembro de 2004. Usou-se, para análise estatística, um estudo com regressão e, para comparações entre as regressões, o procedimento descrito em Snedecor \& Cochran (1989). Obtiveram-se os valores de GDD com a metodologia apresentada por Ometto (1981), comparada com os métodos de Arnold (1959), Snyder (1985) e Dufault (1997); $\mathrm{Tb}$ de $12,9{ }^{\circ} \mathrm{C}$ e TB de $32,4^{\circ} \mathrm{C}$, foram obtidos após comparações entre os métodos: menor desvio padrão em graus-dia e dias, coeficiente de variação em graus-dia, coeficiente de regressão e análise de Kc versus GDD; para ambas as cultivares até 4200 GDD, chegou-se a valores de 0,20, 0,89 e 0,48, para Kc mínimo, máximo e médio, respectivamente.
\end{abstract}

Palavras-chave: café irrigado, manejo de irrigação, clima

\section{Base temperature, crop coefficients and degrees-day for arabic coffee plants in the implantation phase}

\begin{abstract}
This paper had as its purpose the determination of the inferior base temperature - Tb, and superior - TB, for arabic coffee plants. The cultivars "Acaiá Cerrado" (MG-1474) and "Rubi" (MG-1192) were evaluated, associating crop coefficients - Kc, to growing degrees-day - GDD. The trial was carried out in the "Universidade Federal de Lavras" - UFLA, from March 2003 to September 2004. For statistical analysis, a study with regression and comparisons among regressions using the procedure described in Snedecor \& Cochran (1989) were used. The values of GDD were obtained with the methodology presented by Ometto (1981), and were compared to the methods presented by Arnold (1959), Snyder (1985) and Dufault (1997); $\mathrm{Tb}=12.9^{\circ} \mathrm{C}$ and $\mathrm{TB}=32.4{ }^{\circ} \mathrm{C}$ were obtained after comparisons among the methods: least standard deviation in degrees-day and days, coefficient of variation in degrees-day, regression coefficient and analysis of Kc versus GDD were found. For both cultivars, up to $4200 \mathrm{GDD}$, values of $0.20,0.89$ and 0.48 were found, for Kc minimum, maximum and mean, respectively.
\end{abstract}

Key words: irrigated coffee, irrigation management, climate

\footnotetext{
Parte da Tese de Doutorado do primeiro autor apresentada ao Programa de Pós-graduação em Engenharia Agrícola da Universidade Federal de Lavras, financiada com recursos do CNPq e bolsa da CAPES

2 Rua Evaristo Alves, 111, Centro, CEP 37200-000, Lavras, MG. Fone: (35) 3821-5742. E-mail: eplima1@ig.com.br

3 UFLA/DEG, CEP 37200-000 Lavras, MG. Fone: (35) 3829-1664. E-mail: elemos@ufla.br
} 


\section{INTRODUÇÃO}

Grande parte do sucesso de uma agricultura irrigada depende do manejo adequado da irrigação. A irrigação é influenciada sobretudo pelas características físicas dos solos, pelas características da cultura e pelo clima, tornando-se uma das principais ferramentas para a agricultura moderna.

A determinação de lâmina de irrigação através de coeficientes de cultura, Kc, pode ser equivocada se as condições dos locais, em que Kc foi obtido e onde a cultura está implantada, forem diferentes, podendo-se superestimar ou subestimar o consumo de água pela lavoura. A utilização apropriada de variáveis meteorológicas que influenciam o crescimento e o desenvolvimento das plantas permite determinar, com maior precisão, a duração das fases fenológicas das plantas cultivadas. Uma alternativa de caracterizar cada uma dessas fases é a utilização dos graus-dia, que nada mais é que o acúmulo diário da energia. Para definir esta nova forma de acompanhamento das fases da cultura e relacioná-la a momento e lâmina de irrigação, é preciso se estabelecer, de início, a temperatura mínima exigida pelo cafeeiro, em prol do desenvolvimento (temperatura base inferior), e os valores de Kc durante as suas diferentes fases fenológicas. Esta temperatura base representa a menor temperatura abaixo da qual o desenvolvimento da planta é nulo; abaixo desta temperatura a planta não se desenvolve e, se o fizer, será a uma taxa muito reduzida.

Com uma curva de Kc como função de graus-dia de desenvolvimento (GDD) mais dados de evapotranspiração de referência, ETo, e termômetros de máxima e mínima, espera-se que o irrigante ou pesquisador possa determinar, com mais precisão, o momento certo de irrigar e a lâmina mais adequada de irrigação.

Desta forma se objetivou, com o presente trabalho, determinar as temperaturas basais, inferior e superior, e coeficientes de cultura, Kc, de cafeeiros arábica (Coffea arabica) em fase de implantação, de forma a permitir acompanhar o seu desenvolvimento pelo método de graus-dia de desenvolvimento (GDD), associado à exigência de água.

\section{MATERIAL E MÉTODOS}

O trabalho ora apresentado foi conduzido no Departamento de Engenharia (DEG) da Universidade Federal de Lavras (UFLA) em uma área ao lado da estação meteorológica automática, pertencente à rede de plataforma de coleta de dados (PCD) do Instituto Nacional de Pesquisas Espaciais (INPE).

As coordenadas geográficas da referida área, são: $21^{\circ} 14^{\text {' }}$ de latitude Sul $45^{\circ} 00^{\prime}$ longitude Oeste e altitude média de 918,8 m. O solo do experimento é classificado como Latossolo Vermelho Distroférrico Típico, segundo EMBRAPA (1999).

Aplicando-se o método de classificação climática de Köppen, apresentado por Ometto (1981), às Normais Climatológicas de Lavras, Brasil (1992), obtém-se clima Cwa, temperado chuvoso, com inverno seco. A temperatura média anual é $19,4^{\circ} \mathrm{C}$, o total de precipitação anual de $1.530 \mathrm{~mm}$, o total de evaporação anual de $1.034 \mathrm{~mm}$ e a umidade relativa média anual de $76 \%$.
Realizaram-se as regressão relativas ao ensaio com o aplicativo SigmaPlot, versão 9.01 da Jandel Scientific e, para comparações entre as regressões, o procedimento descrito por Snedecor \& Cochran (1989).

O experimento foi conduzido com duas cultivares de cafeeiro (Coffea arábica L.): cultivar Rubi MG-1192 e cultivar Acaiá Cerrado MG-1474, cujo transplantio se deu no decorrer de um ano, a cada mês, a partir de 19 de março de 2003, quando as mudas atingiam 6 a 7 pares de folhas.

Transplantaram-se vinte parcelas de cada cultivar, as quais foram identificadas pela sua data de transplantio e pela cultivar correspondente, e cada parcela era constituída de nove plantas em linha, das quais sete formaram a sua área útil. $\mathrm{O}$ espaçamento de transplantio foi de 3,5 x 0,80 m, para as duas cultivares. Para este trabalho consideraram-se somente cinco datas das dez transplantadas, devido ao fato de que apenas as cinco primeiras datas, D1 a D5 correspondendo, seqüencialmente, a 19/mar/2003, 19/abr/2003, 19/mai/2003, 19/jun/2003 e 19/jul/2003 atingiram, até o período final de coleta de dados, a primeira florada. Para que as mudas se desenvolvessem chegando à florada sem déficit hídrico, as áreas foram irrigadas por gotejamento com uma linha lateral por linha de plantas.

\section{Métodos de determinação da temperatura base}

$\mathrm{Na}$ literatura tem-se encontrado, basicamente, quatros métodos de determinação de temperatura base: menor desvio padrão em graus-dia (SDgd), menor desvio padrão em dias (SDd), coeficiente de variação em graus-dia (CVgd) e coeficiente de regressão (Re). Foram adotadas, nesta pesquisa, as fórmulas matemáticas apresentadas por Yang et al. (1995) e o método indicado por Ometto (1981), cujo propósito foi gerar a temperatura base para o café arábica, desde o transplantio até a primeira florada.

\section{Método do menor desvio padrão, em graus-dia (SDgd)}

Neste método, a temperatura base é selecionada assim que a variação resultante em graus-dia de desenvolvimento (GDD); usando-se uma série de plantios, é minimizada, ou seja, para uma série de temperaturas base aquela que resultar em GDD com o menor desvio padrão será selecionada como temperatura base. Para este método, Yang et al. (1995) apresentam a (Eq. 1), para determinação da temperatura base:

$$
\mathrm{Tb}=\frac{\sum_{\mathrm{i}=1}^{\mathrm{n}}(\mathrm{Ti} \cdot \mathrm{di}) \sum_{\mathrm{i}=1}^{\mathrm{n}} \mathrm{di}-\mathrm{n} \sum_{\mathrm{i}=1}^{\mathrm{n}}\left(\mathrm{di^{2 }} \cdot \mathrm{Ti}\right)}{\left(\sum_{\mathrm{i}=1}^{\mathrm{n}} \mathrm{di}\right)^{2}-\mathrm{n} \sum_{\mathrm{i}=1}^{\mathrm{n}} \mathrm{di} \mathrm{i}^{2}}
$$

em que:

$$
\begin{aligned}
\mathrm{Tb} & \text { - temperatura base } \\
\mathrm{Ti} \text { - } & \text { temperatura média de cada época específica } \\
\mathrm{n} \text { - } & \text { número de épocas de plantio } \\
\mathrm{di} \text { - } & \text { número de dias requerido para se alcançar um } \\
& \text { estágio de desenvolvimento em cada série de } \\
& \text { plantio específica }
\end{aligned}
$$




\section{Método do menor desvio padrão, em dias (SDd)}

Arnold (1959) sugeriu que o uso dessas unidades de calor não considera o erro em unidades de calor como tal, mas, no erro em dias que esta unidade de calor representa. Explorando matematicamente essas afirmações, Yang et al. (1995) apresentam a (Eq. 2), para determinação da temperatura base:

$$
\mathrm{Tb}=\mathrm{T}-\frac{\left(\sum_{\mathrm{i}=1}^{\mathrm{n}} \mathrm{ti} \cdot \mathrm{di}\right)^{2}-\mathrm{n} \sum_{\mathrm{i}=1}^{\mathrm{n}}\left(\mathrm{ti}^{2} \cdot \mathrm{di}^{2}\right)}{\mathrm{n} \sum_{\mathrm{i}=1}^{\mathrm{n}}\left(\mathrm{di} \mathrm{i}^{2} \cdot \mathrm{ti}\right)-\mathrm{n} \sum_{\mathrm{i}=1}^{\mathrm{n}}(\mathrm{ti} \cdot \mathrm{di}) \sum_{\mathrm{i}=1}^{\mathrm{n}} \mathrm{di}}
$$

em que:

$$
\begin{aligned}
\text { Tb - temperatura base } \\
\mathrm{T} \text { - temperatura média para todas as séries de plantio } \\
\mathrm{n} \text { - número de séries de plantio } \\
\mathrm{di} \text { - número de dias requerido para se alcançar um } \\
\\
\quad \text { estágio de desenvolvimento em cada série de } \\
\text { plantio específica } \\
\text { ti - diferença entre a temperatura média para todas } \\
\\
\text { as séries de plantio ( } \mathrm{T} \text { ) e a temperatura média } \\
\text { em uma série específica (TMi) }
\end{aligned}
$$

\section{Método do coeficiente de variação, em graus-dia (CVgd)}

Os métodos descritos acima, SDgd e SDd, oferecem magnitudes absolutas da variação em GDD e em dias mas não mencionam magnitudes relativas da variação; em função disto, Nuttonson (1958) apud Yang et al. (1995), definiu o coeficiente de variação como a relação entre SDd e o número médio de dias para se alcançar um estágio de desenvolvimento (Xd). Os mesmos autores desenvolveram, matematicamente, esses conceitos e apresentaram a seguinte fórmula matemática de temperatura base para este método:

$$
\mathrm{Tb}=\frac{\sum_{\mathrm{i}=1}^{\mathrm{n}}\left(\mathrm{Ti} \cdot \mathrm{di} \mathrm{i}^{2}\right) \sum_{\mathrm{i}=1}^{\mathrm{n}}(\mathrm{Ti} \cdot \mathrm{di})-\sum_{\mathrm{i}=1}^{\mathrm{n}} \mathrm{di} \sum_{\mathrm{i}=1}^{\mathrm{n}}\left(\mathrm{Ti}^{2} \cdot \mathrm{di}^{2}\right)}{\sum_{\mathrm{i}=1}^{\mathrm{n}} \mathrm{di} \mathrm{i}^{2} \sum_{\mathrm{i}=1}^{\mathrm{n}}(\mathrm{Ti} \cdot \mathrm{di})-\sum_{\mathrm{i}=1}^{\mathrm{n}} \mathrm{di} \sum_{\mathrm{i}=1}^{\mathrm{n}}\left(\mathrm{Ti} \cdot \mathrm{di}^{2}\right)}
$$

em que:

$$
\begin{aligned}
& \text { Tb - temperatura base } \\
& \mathrm{Ti} \text { - temperatura média para cada série de plantio } \\
& \mathrm{n} \text { - número de séries de plantio } \\
& \mathrm{di} \text { - número de dias requerido para se alcançar um } \\
& \quad \text { estágio de desenvolvimento em cada série de } \\
& \quad \text { plantio específica }
\end{aligned}
$$

\section{Método do coeficiente de regressão (Re)}

Nos métodos anteriores pode acontecer da temperatura base, que gerou os GDD com a menor variação, ser muito abaixo de zero, devido ao fato dos três métodos anteriores serem empíricos, o que levará, sem dúvida, a uma nova seleção de valores de temperatura base para outro processo interativo de cálculo, dificultando a seleção da temperatura base correta, que gera os GDD ou dias com a menor variação.

Em relação às falhas dos três métodos discutidos acima, Hoover (1955) apud Yang et al. (1995), desenvolveu o méto- do do coeficiente de regressão para calcular a temperatura base a partir dos graus-dia para cada série específica de plantio, desenvolveram esses conceitos e ainda apresentaram a fórmula matemática de temperatura base para este método, Eq. 4.

O modelo estudado envolve a relação entre a temperatura média e GDD por meio de uma regressão linear em que a temperatura média é a variável independente e GDD, a variável dependente.

Teoricamente, se a temperatura selecionada fosse muito alta, o coeficiente de regressão no modelo seria positivo; caso contrário, negativo, quando o coeficiente de regressão era zero, considerava-se que a temperatura selecionada era também a temperatura base, qual seja:

$$
\mathrm{Tb}=\frac{\sum_{\mathrm{i}=1}^{\mathrm{n}} \mathrm{Ti} \sum_{\mathrm{i}=1}^{\mathrm{n}}(\mathrm{di} \cdot \mathrm{Ti})-\mathrm{n} \sum_{\mathrm{i}=1}^{\mathrm{n}}\left(\mathrm{di} \cdot \mathrm{Ti}^{2}\right)}{\sum_{\mathrm{i}=1}^{\mathrm{n}} \mathrm{di} \sum_{\mathrm{i}=1}^{\mathrm{n}} \mathrm{Ti}-\mathrm{n} \sum_{\mathrm{i}=1}^{\mathrm{n}}(\mathrm{di} \cdot \mathrm{Ti})}
$$

em que:

$$
\begin{aligned}
& \text { Tb - temperatura base } \\
& \mathrm{Ti} \text { - temperatura média para cada série específica } \\
& \mathrm{n} \text { - } \text { número de séries de plantio } \\
& \mathrm{di} \text { - } \text { número de dias requeridos para se alcançar um } \\
& \text { estágio de desenvolvimento em cada época es- } \\
& \text { pecífica }
\end{aligned}
$$

\section{Método apresentado por Ometto (1981)}

O uso deste método leva em consideração cinco casos distintos de determinação de graus-dia de desenvolvimento para a determinação das temperaturas base, inferior (Tb) e superior (TB).

Para calcular a temperatura base inferior (Tb), estabeleceu-se um intervalo entre dois períodos distintos de transplantio, em que não houve possibilidade de ocorrerem temperaturas suficientemente elevadas e que poderiam alcançar a temperatura base superior (TB). O intervalo escolhido foi no inverno-primavera, considerando-se as datas de transplantios D4 (Período 1) e D5 (Período 2).

Atingindo a primeira florada, os números de graus-dia tendem a ser semelhantes; assim, considera-se que o número de dias para um período $\left(\mathrm{N}_{1}\right)$ está para o outro $\left(\mathrm{N}_{2}\right)$, assim como as ocorrências da temperatura mínima $\left(\mathrm{Tm}_{1}\right)$ e máxima $\left(\mathrm{TM}_{1}\right)$ em um período, estão para as do outro período $\left(\mathrm{Tm}_{2}\right.$ e $\left.\mathrm{TM}_{2}\right)$, respectivamente.

Para determinação da temperatura base superior (TB), segue-se o mesmo desenvolvimento de determinação da temperatura base inferior $(\mathrm{Tb})$, porém se deve considerar o período de temperaturas elevadas no processo de cálculo; neste caso particular, o valor obtido de temperatura base inferior entrará no cálculo da temperatura base superior.

\section{Determinação de graus-dia de desenvolvimento (GDD)}

Para o cálculo de graus-dia foram usadas temperaturas, máxima e mínima do dia, coletadas na estação meteorológica automática da rede de plataformas de coleta de dados (PCD) do Instituto Nacional de Pesquisas Espaciais (INPE), que se encontra ao lado da área experimental. Os valores 
usados de temperaturas base inferior e superior foram determinados em item específico desta pesquisa e os métodos utilizados para determinação de graus-dia de desenvolvimento (GDD), para o período de análise, foram: método Residual, segundo Arnold (1959), método Ometto (1981), método Snyder (1985) e método Dufault (1997).

\section{Método do menor desvio padrão, em dias}

Este método também é conhecido como método residual, apresentado por Arnold (1959) segundo a equação:

$$
\mathrm{GD}=\frac{\mathrm{TM}+\mathrm{Tm}}{2}-\mathrm{Tb}
$$

em que:

$$
\begin{aligned}
& \mathrm{GD} \text { - graus-dia, }{ }^{\circ} \mathrm{C} \\
& \mathrm{TM} \text { - temperatura máxima do dia, }{ }^{\circ} \mathrm{C} \\
& \mathrm{Tm} \text { - temperatura mínima do dia, }{ }^{\circ} \mathrm{C} \\
& \mathrm{Tb} \text { - temperatura base inferior, }{ }^{\circ} \mathrm{C}
\end{aligned}
$$

\section{Método apresentado por Ometto (1981)}

Segundo Ometto (1981), existe um valor de energia mínima que aciona os dispositivos metabólicos da planta, chamado temperatura base inferior (Tb), em que somente estados energéticos acima deste limite são propícios ao crescimento e ao desenvolvimento do vegetal estudado.

O nível energético máximo, acima do qual a planta estanca suas atividades metabólicas, é chamado temperatura base superior (TB) e compreende o limite superior de suporte energético para aquela planta.

O intervalo de tempo durante aquele dia em que a energia do meio ficou abaixo do valor mínimo necessário à planta, é desprezado, pois ela estava com suas funções vitais praticamente estacionadas; o mesmo acontece quando a energia do meio se encontra acima do valor máximo admissível para aquela planta. O “quantum” de energia que realmente pode interagir com a planta, trazendo estímulo aos processos metabólicos, fica entre os níveis mínimo e máximo basais; portanto, os valores de temperatura do ar inferiores ao mínimo basal e os superiores ao máximo basal, são retirados do cálculo de graus-dia por se constituírem em valores deficitários, no caso do mínimo, e excessivo, no caso do máximo.

Desta forma, Ometto (1981) apresenta cinco alternativas de cálculo do "acúmulo de energia” ou graus-dia, sempre considerando o tempo unitário de um dia.

$$
\begin{aligned}
& \text { Caso 1: } \mathrm{TB}>\mathrm{TM}>\mathrm{Tm}>\mathrm{Tb} \\
& \mathrm{GD}=\frac{\mathrm{TM}-\mathrm{Tm}}{2}+\mathrm{Tm}-\mathrm{Tb}
\end{aligned}
$$

Caso 2: $\mathrm{TB}>\mathrm{TM}>\mathrm{Tb}>\mathrm{Tm}$

$$
\mathrm{GD}=\frac{(\mathrm{TM}-\mathrm{Tb})^{2}}{2 \cdot(\mathrm{TM}-\mathrm{Tm})}
$$

Caso 3: $\mathrm{TB}>\mathrm{Tb}>\mathrm{TM}>\mathrm{Tm}$

$\mathrm{GD}=0$
Caso 4: $\mathrm{TM}>\mathrm{TB}>\mathrm{Tm}>\mathrm{Tb}$

$$
\mathrm{GD}=\frac{2 \cdot(\mathrm{TM}-\mathrm{Tm}) \cdot(\mathrm{Tm}-\mathrm{Tb})+(\mathrm{TM}-\mathrm{Tm})^{2}-(\mathrm{TM}-\mathrm{TB})}{2 \cdot(\mathrm{TM}-\mathrm{Tm})}
$$

Caso 5: $\mathrm{TM}>\mathrm{TB}>\mathrm{Tb}>\mathrm{Tm}$

$$
\mathrm{GD}=\frac{1}{2} \cdot \frac{(\mathrm{TM}-\mathrm{Tb})^{2}-(\mathrm{TM}-\mathrm{TB})^{2}}{\mathrm{TM}-\mathrm{Tm}}
$$

em que:

$$
\begin{aligned}
& \mathrm{GD} \text { - graus-dia, }{ }^{\circ} \mathrm{C} \\
& \mathrm{TM} \text { - temperatura máxima do dia, }{ }^{\circ} \mathrm{C} \\
& \mathrm{Tm} \text { - temperatura mínima do dia, }{ }^{\circ} \mathrm{C} \\
& \mathrm{Tb} \text { - temperatura base inferior, }{ }^{\circ} \mathrm{C} \\
& \mathrm{TB} \text { - temperatura base superior, }{ }^{\circ} \mathrm{C}
\end{aligned}
$$

\section{Método apresentado por Snyder (1985)}

Segundo Snyder (1985), os graus-dia são determinados considerando-se quatro casos distintos:

Caso 1: $\mathrm{TB}>\mathrm{TM}>\mathrm{Tm}>\mathrm{Tb}$

Para este caso, os valores de graus-dia são determinados de acordo com a (Eq. 5), de Arnold (1959).

Caso 2: $\mathrm{TB}>\mathrm{TM}>\mathrm{Tb}>\mathrm{Tm}$

Para este caso, os valores de graus-dia serão definidos conforme a (Eq. 11), sendo as variáveis $\theta$, M e W determinadas através das (Eq. 12, 13 e 14), respectivamente.

$$
\begin{aligned}
& \mathrm{GD}=\frac{(\mathrm{M}-\mathrm{Tb}) \cdot(\pi / 2-\theta)+\mathrm{W} \cdot \cos (\theta)}{\pi} \\
& \theta=\operatorname{arcsen}\left(\frac{\mathrm{Tb}-\mathrm{M}}{\mathrm{W}}\right) \\
& \mathrm{M}=\frac{\operatorname{Tmax}+\mathrm{Tmin}}{2} \\
& \mathrm{~W}=\frac{\operatorname{Tmax}-\mathrm{Tmin}}{2}
\end{aligned}
$$

Caso 3: $\mathrm{TM}>\mathrm{TB}>\mathrm{Tm}>\mathrm{Tb}$

Aqui, os valores de graus-dia serão determinados segundo a (Eq. 15), sendo a variável $\phi$ mensurada utilizando-se a Eq. 16.

$$
\begin{aligned}
& \mathrm{GD}=\mathrm{M}-\mathrm{Tb}-\frac{(\mathrm{M}-\mathrm{TB}) \cdot(\pi / 2-\phi)+\mathrm{W} \cdot \cos (\phi)}{\pi} \\
& \phi=\operatorname{arcsen}\left(\frac{\mathrm{TB}-\mathrm{M}}{\mathrm{W}}\right)
\end{aligned}
$$

Caso 4: $\mathrm{TM}>\mathrm{TB}>\mathrm{Tb}>\mathrm{Tm}$

Para este caso, os valores de graus-dia serão determinados de acordo com a Eq. 17.

$$
\mathrm{GD}=\frac{(\mathrm{M}-\mathrm{Tb}) \cdot(\pi / 2-\theta)+\mathrm{W} \cdot \cos (\theta)}{\pi}-\frac{(\mathrm{M}-\mathrm{TB}) \cdot(\pi / 2-\phi)+\mathrm{W} \cdot \cos (\phi)}{\pi}
$$

em que: 
GD - graus-dia, ${ }^{\circ} \mathrm{C}$

$\mathrm{TM}$ - temperatura máxima do dia, ${ }^{\circ} \mathrm{C}$

Tm - temperatura mínima do dia, ${ }^{\circ} \mathrm{C}$

$\mathrm{Tb}$ - temperatura base inferior, ${ }^{\circ} \mathrm{C}$

$\mathrm{TB}$ - temperatura base superior, ${ }^{\circ} \mathrm{C}$

$\mathrm{W}, \theta$ e $\phi-$ variáveis do modelo

\section{Método apresentado por Dufault (1997)}

Os graus-dia calculados por este método podem ser feitos por meio de dois casos distintos.

\section{Caso 1: $\mathrm{TM} \leq \mathrm{TB}$}

Usado quando a temperatura máxima do dia não exceder a temperatura base superior, caso em que basta subtrair, da temperatura média diária, o valor da temperatura base inferior (Eq. 5), Equação de Arnold (1959).

\section{Caso 2: $\mathrm{TM}>\mathrm{TB}$}

Indicado quando a temperatura máxima do dia exceder a temperatura base superior, o que faz com que ocorra um crescimento reduzido associado a altas temperaturas (Eq. 18); neste caso, será introduzido um fator de ajuste para a temperatura máxima do dia, de acordo com a seguinte equação:

$$
\begin{aligned}
& \mathrm{GD}=\frac{\mathrm{T}_{\mathrm{ADJ}-\max }+\mathrm{Tm}}{2}-\mathrm{Tb} \\
& \mathrm{T}_{\mathrm{AJD}-\max }=\mathrm{TB}-(\mathrm{TM}-\mathrm{TB})
\end{aligned}
$$

em que:

$$
\text { GD - graus-dia, }{ }^{\circ} \mathrm{C}
$$

$\mathrm{T}_{\mathrm{ADJ}-\max }$ - temperatura máxima diária ajustada, ${ }^{\circ} \mathrm{C}$

$\mathrm{Tb}$ - temperatura base inferior, ${ }^{\circ} \mathrm{C}$

$\mathrm{TB}$ - temperatura base superior, ${ }^{\circ} \mathrm{C}$

$\mathrm{TM}$ - temperatura máxima do dia, ${ }^{\circ} \mathrm{C}$

$\mathrm{Tm}$ - temperatura mínima do dia, ${ }^{\circ} \mathrm{C}$

\section{RESULTADOS E DISCUSSÃO}

\section{Temperatura base}

Os cincos casos de determinação de GDD apresentados por Ometto (1981) foram trabalhados matematicamente com auxílio do Software da Mathsoft Engineering \& Education, Inc., Mathcad, version 12.0, para a determinação das equações de temperatura base.

$$
\begin{aligned}
& \text { Caso 1: } \mathrm{TB}>\mathrm{TM}>\mathrm{Tm}>\mathrm{Tb} \\
& \mathrm{Tb}=-\frac{1}{2} \cdot \frac{\mathrm{N}_{1} \cdot \mathrm{TM}_{1}+\mathrm{N}_{1} \cdot \mathrm{Tm}_{1}-\mathrm{N}_{2} \cdot \mathrm{TM}_{2}-\mathrm{N}_{2} \cdot \mathrm{Tm}_{2}}{\mathrm{~N}_{2}-\mathrm{N}_{1}}
\end{aligned}
$$

Caso 2: $\mathrm{TB}>\mathrm{TM}>\mathrm{Tb} \geq \mathrm{Tm}$

Neste caso, deve-se agrupar as (Eq. 21, 22, 23 e 24) para se obter a equação de temperatura base inferior, que está representada na Eq. 25.

$$
\begin{aligned}
& \mathrm{A}=\mathrm{N}_{2} \cdot \mathrm{TM}_{1} \cdot \mathrm{TM}_{2}-\mathrm{N}_{2} \cdot \mathrm{TM}_{2} \cdot \mathrm{Tm}_{1} \\
& \mathrm{~B}=-\mathrm{N}_{1} \cdot \mathrm{TM}_{1} \cdot \mathrm{TM}_{2}+\mathrm{N}_{1} \cdot \mathrm{TM}_{1} \cdot \mathrm{Tm}_{2}
\end{aligned}
$$

$$
\begin{aligned}
& \mathrm{C}=-\mathrm{N}_{2} \cdot \mathrm{N}_{1} \cdot\left(-\mathrm{TM}_{2}+\mathrm{Tm}_{2}\right) \cdot\left(\mathrm{TM}_{1}-\mathrm{Tm}_{1}\right) \cdot\left(\mathrm{TM}_{1}-\mathrm{TM}_{2}\right)^{2} \\
& \mathrm{D}=-\mathrm{N}_{1} \cdot \mathrm{TM}_{2}+\mathrm{N}_{1} \cdot \mathrm{Tm}_{2}+\mathrm{N}_{2} \cdot \mathrm{TM}_{1}-\mathrm{N}_{2} \cdot \mathrm{Tm}_{1} \\
& \mathrm{~Tb}=\frac{\mathrm{A}+\mathrm{B} \pm \sqrt{\mathrm{C}}}{\mathrm{D}}
\end{aligned}
$$

Verifica-se, pela (Eq. 25), que é possível obter dois valores de Tb; considera-se, para efeito de cálculo, o menor deles, desde que seja positivo.

\section{Caso 03: $\mathrm{TB}>\mathrm{Tb}>\mathrm{TM}>\mathrm{Tm}$}

Ocorre quando as temperaturas base inferior e base superior (Tb, TB) da planta estiverem acima da temperatura máxima do ar (TM); neste caso, a energia à disposição da planta estará abaixo do valor mínimo necessário a ela e a energia acumulada, expressa em graus-dia é, portanto, nula.

Caso 04: $\mathrm{TM}>\mathrm{TB}>\mathrm{Tm}>\mathrm{Tb}$

Neste caso, deve-se agrupar as (Eq. 26, 27, 28, 29, 30, 31 e 32) para se obter a equação de temperatura base inferior, que está representada na Eq. 33.

$$
\begin{aligned}
& \mathrm{A}=\mathrm{N}_{1} \cdot \mathrm{Tm}_{1}^{2} \cdot \mathrm{TM}_{2}-\mathrm{N}_{1} \cdot \mathrm{Tm}_{1}^{2} \cdot \mathrm{Tm}_{2} \\
& \mathrm{~B}=-2 \cdot \mathrm{N}_{1} \cdot \mathrm{TM}_{1} \cdot \mathrm{TB} \cdot \mathrm{TM}_{2}+2 \cdot \mathrm{N}_{1} \cdot \mathrm{TM}_{1} \cdot \mathrm{TB} \cdot \mathrm{Tm}_{2} \\
& \mathrm{C}=\mathrm{N}_{1} \cdot \mathrm{TB}^{2} \cdot \mathrm{TM}_{2}-\mathrm{N}_{1} \cdot \mathrm{TB}^{2} \cdot \mathrm{Tm}_{2} \\
& \mathrm{D}=-\mathrm{N}_{2} \cdot \mathrm{Tm}_{2}^{2} \cdot \mathrm{TM}_{1}+\mathrm{N}_{2} \cdot \mathrm{Tm}_{2}^{2} \cdot \mathrm{Tm}_{1} \\
& \mathrm{E}=2 \cdot \mathrm{N}_{2} \cdot \mathrm{TM}_{1} \cdot \mathrm{TB}^{2} \mathrm{TM}_{2}-2 \cdot \mathrm{N}_{2} \cdot \mathrm{TM}_{2} \cdot \mathrm{TB} \cdot \mathrm{Tm}_{1} \\
& \mathrm{~F}=-\mathrm{N}_{2} \cdot \mathrm{TB}^{2} \cdot \mathrm{TM}_{1}+\mathrm{N}_{2} \cdot \mathrm{TB}^{2} \cdot \mathrm{Tm}_{1} \\
& \mathrm{G}=\left(-\mathrm{TM}_{1}+\mathrm{Tm}_{1}\right) \cdot\left(\mathrm{TM}_{2}-\mathrm{Tm}_{2}\right) \cdot\left(\mathrm{N}_{1}-\mathrm{N}_{2}\right) \\
& \mathrm{Tb}=\frac{1}{2} \cdot \frac{\mathrm{A}+\mathrm{B}+\mathrm{C}+\mathrm{D}+\mathrm{E}+\mathrm{F}}{\mathrm{G}}
\end{aligned}
$$

Uma vez conhecido o valor de Tb, basta usá-lo quando solicitado, nas (Eq. 34 a 43) que, agrupadas, fornecem o valor de TB representado na Eq. 44.

$$
\begin{aligned}
& \mathrm{A}=\mathrm{N}_{2} \cdot \mathrm{TM}_{1} \cdot \mathrm{TM}_{2}-\mathrm{N}_{2} \cdot \mathrm{TM}_{2} \cdot \mathrm{Tm}_{1} \\
& \mathrm{~B}=-\mathrm{N}_{1} \cdot \mathrm{TM}_{1} \cdot \mathrm{TM}_{2}+\mathrm{N}_{1} \cdot \mathrm{TM}_{1} \cdot \mathrm{Tm}_{2} \\
& \mathrm{C}=\left(-\mathrm{TM}_{1}+\mathrm{Tm}_{1}\right) \cdot\left(-\mathrm{TM}_{2}+\mathrm{Tm}_{2}\right) \\
& \mathrm{D}=\mathrm{N}_{1} \cdot \mathrm{Tm}_{2}{ }^{2} \cdot \mathrm{N}_{2}-2 \cdot \mathrm{Tm}_{2} \cdot \mathrm{N}_{1} \cdot \mathrm{N}_{2} \cdot \mathrm{Tb}-\mathrm{Tm}_{2} \cdot \mathrm{Tm}_{1} \cdot \mathrm{N}_{1}{ }^{2} \\
& \mathrm{E}=-\mathrm{Tm}_{2} \cdot \mathrm{TM}_{1} \cdot \mathrm{N}_{1}{ }^{2}+\mathrm{N}_{2}{ }^{2} \cdot \mathrm{TM}_{1} \cdot \mathrm{Tm}_{2}-\mathrm{Tm}_{1} \cdot \mathrm{N}_{2}{ }^{2} \cdot \mathrm{Tm}_{2}+2 \cdot \mathrm{Tm}_{2} \cdot \mathrm{N}_{1}^{2} \cdot \mathrm{Tb} \\
& \mathrm{F}=\mathrm{Tm}_{1} \cdot \mathrm{N}_{1}{ }^{2} \cdot \mathrm{TM}_{2}-2 \cdot \mathrm{N}_{2}{ }^{2} \cdot \mathrm{TM}_{1} \cdot \mathrm{Tb}-2 \cdot \mathrm{N}_{1}{ }^{2} \cdot \mathrm{TM}_{2} \cdot \mathrm{Tb} \\
& \mathrm{G}=\mathrm{TM}_{1} \cdot \mathrm{N}_{1}^{2} \cdot \mathrm{TM}_{2}+\mathrm{Tm}_{1}^{2} \cdot \mathrm{N}_{2} \cdot \mathrm{N}_{1}-2 \cdot \mathrm{Tm}_{1} \cdot \mathrm{N}_{2} \cdot \mathrm{N}_{1} \cdot \mathrm{Tb} \\
& \mathrm{H}=2 \cdot \mathrm{Tm}_{1} \cdot \mathrm{N}_{2}{ }^{2} \cdot \mathrm{Tb}+2 \cdot \mathrm{N}_{1} \cdot \mathrm{TM}_{2} \cdot \mathrm{N}_{2} \cdot \mathrm{Tb}+2 \cdot \mathrm{N}_{2} \cdot \mathrm{TM}_{1} \cdot \mathrm{N}_{1} \cdot \mathrm{Tb} \\
& \mathrm{I}=-2 \mathrm{~N}_{2} \cdot \mathrm{TM}_{1} \cdot \mathrm{N}_{1} \cdot \mathrm{TM}_{2}+\mathrm{N}_{2}^{2} \cdot \mathrm{TM}_{1} \cdot \mathrm{TM}_{2}-\mathrm{Tm}_{1} \cdot \mathrm{N}_{2}^{2} \cdot \mathrm{TM}_{2} \\
& \mathrm{~J}=-\mathrm{N}_{1} \cdot \mathrm{TM}_{2}+\mathrm{N}_{1} \cdot \mathrm{Tm}_{2}+\mathrm{N}_{2} \cdot \mathrm{TM}_{1}-\mathrm{N}_{2} \cdot \mathrm{Tm}_{1} \\
& \mathrm{~TB}=\frac{\mathrm{A}+\mathrm{B} \pm \sqrt{\mathrm{C} \cdot(\mathrm{D}+\mathrm{E}+\mathrm{F}+\mathrm{G}+\mathrm{H}+\mathrm{I})}}{\mathrm{J}}
\end{aligned}
$$

Verifica-se, pela (Eq. 44), que é possível se obter dois 
valores de TB; considerou-se, para efeito de cálculo, o maior deles.

Caso 05: $\mathrm{TM}>\mathrm{TB}>\mathrm{Tb}>\mathrm{Tm}$

Neste caso, deve-se agrupar as (Eq. 45, 46, 47 e 48) para se obter a equação de temperatura base inferior, que está representada na Eq. 49.

$$
\begin{aligned}
& \mathrm{A}=2 \cdot \mathrm{N}_{2} \cdot \mathrm{TM}_{2} \cdot \mathrm{Tm}_{1}-2 \cdot \mathrm{N}_{2} \cdot \mathrm{TM}_{1} \cdot \mathrm{TM}_{2}+2 \cdot \mathrm{N}_{1} \cdot \mathrm{TM}_{1} \cdot \mathrm{TM}_{2} \\
& \mathrm{~B}=-2 \cdot \mathrm{N}_{1} \cdot \mathrm{TM}_{1} \cdot \mathrm{Tm}_{2}-\mathrm{N}_{1} \cdot \mathrm{TB} \cdot \mathrm{TM}_{2}+\mathrm{N}_{1} \cdot \mathrm{TB} \cdot \mathrm{Tm}_{2} \\
& \mathrm{C}=\mathrm{N}_{2} \cdot \mathrm{TB} \cdot \mathrm{TM}_{1}-\mathrm{N}_{2} \cdot \mathrm{TB} \cdot \mathrm{Tm}_{1} \\
& \mathrm{D}=\mathrm{N}_{1} \cdot \mathrm{TM}_{2}-\mathrm{N}_{1} \cdot \mathrm{Tm}_{2}-\mathrm{N}_{2} \cdot \mathrm{TM}_{1}+\mathrm{N}_{2} \cdot \mathrm{Tm}_{1} \\
& \mathrm{~Tb}=\frac{\mathrm{A}+\mathrm{B}+\mathrm{C}}{\mathrm{D}}
\end{aligned}
$$

Uma vez conhecido o valor de Tb, basta usá-lo nas (Eq. 50 a 53) que, agrupadas, fornecem o valor de TB representado na Eq. 54.

$$
\begin{aligned}
& \mathrm{A}=-\mathrm{N}_{1} \cdot \mathrm{Tb} \cdot \mathrm{TM}_{2}+\mathrm{N}_{1} \cdot \mathrm{Tb} \cdot \mathrm{Tm}_{2}+\mathrm{N}_{2} \cdot \mathrm{TM}_{1} \cdot \mathrm{Tb} \\
& \mathrm{B}=-\mathrm{N}_{2} \cdot \mathrm{Tm}_{1} \cdot \mathrm{Tb}-2 \cdot \mathrm{N}_{2} \cdot \mathrm{TM}_{1} \cdot \mathrm{TM}_{2}+2 \cdot \mathrm{N}_{2} \cdot \mathrm{TM}_{2} \cdot \mathrm{Tm}_{1} \\
& \mathrm{C}=2 \cdot \mathrm{N}_{1} \cdot \mathrm{TM}_{1} \cdot \mathrm{TM}_{2}-2 \cdot \mathrm{N}_{1} \cdot \mathrm{TM}_{1} \cdot \mathrm{Tm}_{2} \\
& \mathrm{D}=\mathrm{N}_{1} \cdot \mathrm{TM}_{2}-\mathrm{N}_{1} \cdot \mathrm{Tm}_{2}-\mathrm{N}_{2} \cdot \mathrm{TM}_{1}+\mathrm{N}_{2} \cdot \mathrm{Tm}_{1} \\
& \mathrm{~TB}=\frac{(\mathrm{A}+\mathrm{B}+\mathrm{C})}{\mathrm{D}}
\end{aligned}
$$

Com as temperaturas máximas e mínimas coletadas entre19/mar/2003 a 26/set/2004, geraram-se as temperaturas médias diárias, cujos valores foram usados na parametrização e no estudo dos modelos de determinação da temperatura base.

Os valores de temperatura base inferior ( $\mathrm{Tb}$ ) encontrados, foram de $12,9^{\circ} \mathrm{C}$, pelo método apresentado por Ometto (1981), $14,8{ }^{\circ} \mathrm{C}$ para os métodos do menor desvio padrão em dias (SDd), método do coeficiente de regressão (Re) e coeficiente de variação em graus-dias (CVgd) e $15,4^{\circ} \mathrm{C}$, pelo método do menor desvio padrão em graus-dia (SDgd). A temperatura base superior (TB) foi determinada apenas pelo método de Ometto (1981), encontrando-se o valor de $32,4^{\circ} \mathrm{C}$.

No que se refere à cultura do cafeeiro, não se conhecem muitos estudos para a determinação das temperaturas base inferior (Tb) e superior (TB); entretanto e de acordo com Iaffe et al. (2001), a Tb foi, para o café, de $11^{\circ} \mathrm{C}$ para a região de Campinas, SP.

Coelho (2004), na determinação do coeficiente de cultura para duas variedades de cafeeiro (Coffea arabica L.) em Lavras, MG, associado a graus-dia, adotou, com base na em revisão de literatura, valores de Tb e TB de, respectivamente, $13^{\circ} \mathrm{C}$ e $34^{\circ} \mathrm{C}$. Esses valores, independente do local de determinação e das condições climáticas, apresentaram grande semelhança aos valores obtidos por meio da metodologia de Ometto (1981), no que se refere aos limites inferior $\left(12,9^{\circ} \mathrm{C}\right)$ e superior $\left(32,4^{\circ} \mathrm{C}\right)$ de temperatura base.
A temperatura base depende, além das condições experimentais, dos métodos empregados em sua determinação mas a escolha do valor de Tb igual a $12,9{ }^{\circ} \mathrm{C}$ e TB igual a $32,4{ }^{\circ} \mathrm{C}$, entre aqueles determinados neste trabalho, foi em função de um estudo associando coeficiente de cultura (Kc) a grausdia de desenvolvimento (GDD), que será apresentado e discutido a seguir.

\section{Coeficiente de cultura (Kc) em função de graus-dia de desenvolvimento (GDD)}

Para as cultivares Acaiá Cerrado e Rubi, as curvas obtidas quando se usaram, no cálculo de graus-dia, as Tb de $14,8^{\circ} \mathrm{C}$ e $15,4{ }^{\circ} \mathrm{C}$ apresentam, em seu início, valores decrescentes de Kc, inviabilizando-os na representação da curva de Kc em função de GDD; com o usou da Tb de $12,9^{\circ} \mathrm{C}$, obtiveram-se melhores resultados; este valor será o usado no cálculo de GDD.

Para a cultivar Acaiá Cerrado, todos os modelos de determinação de graus-dia foram altamente significativos, com $\mathrm{r}^{2}$ variando de 0,92 a 0,96 , porém para a cultivar Rubi os mesmos modelos de determinação de graus-dia não foram significativos, mostrando uma resposta de Kc melhor em função de GDD, para a cultivar Acaiá Cerrado.

Analisando-se as equações de regressão de Kc versus GDD após linearização e se utilizando o procedimento de Snedecor \& Cochran (1989), conclui-se que os modelos não se diferenciaram estatisticamente; pode-se, então, inferir que o Kc, em função de GDD, não indicou diferenças para os dados associados de ambas as cultivares quando obtidos para cada método de determinação de GDD; logo, os valores de Kc e GDD foram agrupados, resultando em novas curvas (Figura 1).

Nesta análise, todos os métodos de determinação de GDD foram altamente significativos. Como o propósito é procurar uma única equação que atenda a ambas as cultivares, na análise apresentada a seguir se leva em consideração as novas equações obtidas.

Por se tratar de duas cultivares com estruturas diferentes e o Kc ser um indicador de grande significado físico e biológico, esses resultados podem ser explicados segundo Allen et al. (1994), Arruda et al. (2000) e Wright (1982), quando afirmam que os valores de Kc dependem da área foliar, arquitetura da planta (parte aérea e sistema radicular), cobertura vegetal e da superfície transpirante.

Nesta análise, todos os métodos de determinação de GDD foram altamente significativos porém o que melhor ajustou Kc a valores de GDD, foi o de Ometto (1981) (Figura 1B), que proporcionou um $\mathrm{r}^{2}$ de 0,75 ; desta forma, serão indicados, para as duas cultivares, as temperaturas base inferior e superior de $12,9^{\circ} \mathrm{C}$ e $32,4^{\circ} \mathrm{C}$, respectivamente, e o método de determinação de graus-dia também apresentado por Ometto (1981).

Com os valores indicados de Tb e TB e o método de determinação de GDD, chegou-se ao ajuste combinado de Kc para as cultivares Acaiá Cerrado e Rubi, até 4200 GDD, conforme (Figura 2), sendo os valores 0,20, 0,89 e 0,48, Kc mínimo, máximo e médio, respectivamente.

Referidos valores estão próximos aos encontrados por 


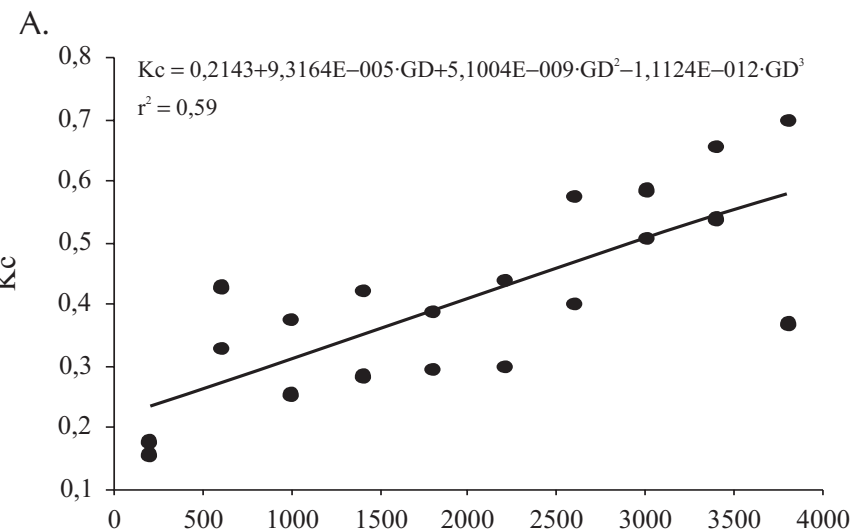

B.

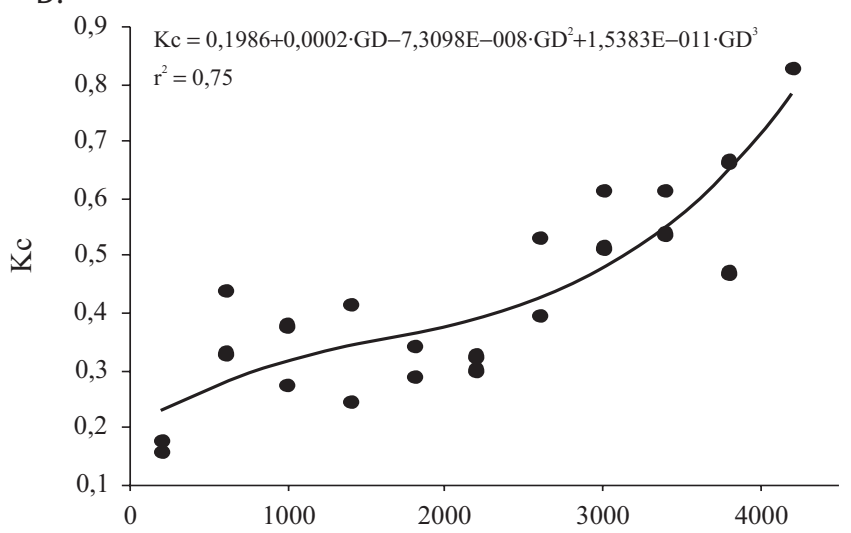

C.
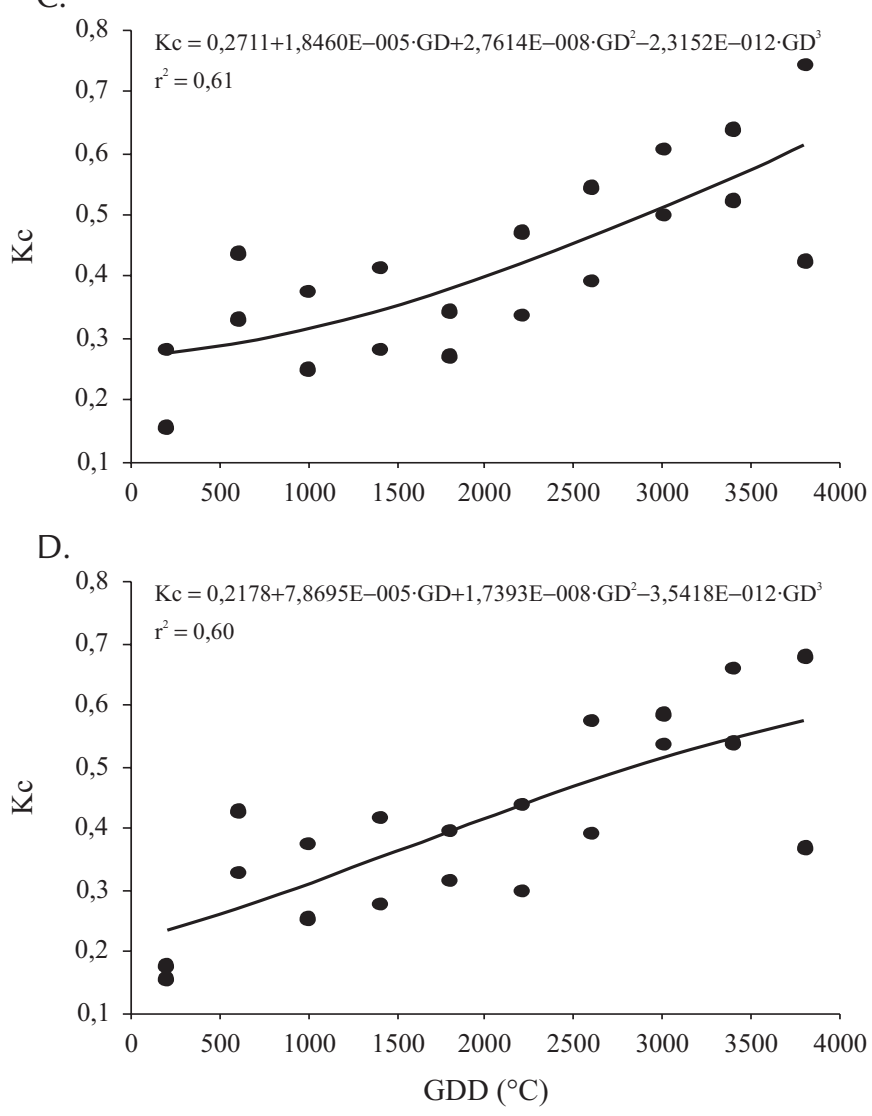

Figura 1. Curvas de Kc em função de graus-dia de desenvolvimento (GDD), considerando-se os dados agrupados das cultivares Acaiá Cerrado e Rubi, e os métodos: (A) residual, (B) Ometto (1981), (C) Snyder (1985) e (D) Dufault (1997). Lavras, MG. 2006

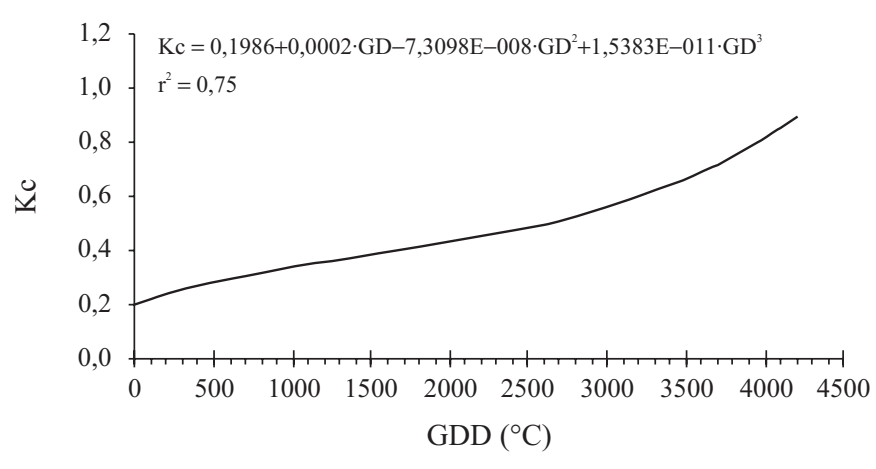

Figura 2. Coeficientes de cultura em função de graus-dia de desenvolvimento (GDD), para café arábica, cultivares Acaiá Cerrado e Rubi, determinados entre o transplantio e a floração, nas condições climáticas de Lavras, MG

Oliveira (2003), 0,44 a 0,87, com valor médio de 0,51 para uma cultura de café recepado para o período de junho a outubro, na região de Lavras, MG; no entanto, os Kc encontrados diferem um pouco dos determinados por Arruda et al. (2000), que ficaram entre 0,73 e 0,75 nos primeiros anos de idade da planta, por Gutiérrez \& Meinzer (1994), que sugerem 0,58 para cafeeiros com 1 ano de plantio, e por Clowes (1984), que considerou um valor médio de 0,6 para todas as fases fenológicas do cafeeiro.

Percebe-se certa variação dos resultados encontrados em relação às sugestões e indicações verificadas na literatura. Conforme Carr (2001) existem poucas informações sobre o consumo hídrico das culturas e os métodos atuais de estudo desse parâmetro; para fins de irrigação, são imprecisos e sujeitos a erros devendo-se testar a sua validade.

\section{CONCLUSÕES}

1. As temperaturas base inferior e superior, para as cultivares Acaiá Cerrado e Rubi, são de 12,9 e $32,4^{\circ} \mathrm{C}$, respectivamente, do transplantio até a primeira florada.

2. O método de determinação de graus-dia apresentado por Ometto (1981) foi o que melhor caracterizou a constante térmica para as cultivares Acaiá Cerrado e Rubi, desde o transplantio até a primeira florada.

3. Os coeficientes de cultura se ajustam satisfatoriamente a graus-dia de desenvolvimento por meio de uma regressão polinomial de $3^{\circ}$ grau.

\section{LITERATURA CITADA}

Allen, R. G.; Smith, M.; Perrier, A.; Pereira, L. S. An update for the definition of reference evapotranspiration. International Commission on Irrigation and Drainage, v.43, n.2, p.93, 1994.

Arnold, C. Y. The determination and significance of the base temperature in a linear heat unit system. Proceedings of the American Society for Horticultural Science, v.74, p.430-445, 1959. 
Arruda, F. B.; Iaffe, A.; Sakai, E.; Calheiros, R. O. Resultados anuais do coeficiente de cultura do cafeeiro em um ensaio em Pindorama/SP. In: Simpósio de Pesquisa dos Cafés do Brasil, 1, 2000, Poços de Caldas. Resumos... Brasília: Embrapa Café e Minasplan, 2000. v.2, p.790-793.

Brasil. Ministério da Agricultura e Reforma Agrária. Normais Climatológicas: 1961-1990. Brasília: SPI/EMBRAPA, 1992. 84p.

Carr, M. K. V. The water relations and irrigation requeriments of coffe. Experimental Agriculturae, v.37, n.1, p.1-36, 2001.

Clowes, M. S. J. Drip irrigation on coffee. Zimbabwe Agricultural Journal, v.81, n.6, p.215-219, 1984.

Coelho, M. R. Coeficiente de cultura (kc) e desenvolvimento inicial de duas variedades de cafeeiro (Coffea arábica L.) associados à graus-dia. Lavras: UFLA, 2004. 75p. Dissertação Mestrado

Dufault, R. J. Determining heat unit requirements for broccoli in coastal South Carolina. Journal of the American Society for Horticultural Science, v.122, n.2, p.169-174, 1997.

EMBRAPA - Empresa Brasileira de Pesquisa Agropecuária. Centro Nacional de Pesquisa de Solos. Sistema brasileiro de classificação de solos. Rio de Janeiro: Embrapa Solos, 1999. 412p.
Gutiérrez, M. V.; Meinzer, F. C. Estimating water use and irrigation requirements of coffee in Hawaii. Journal of the American Society for Horticultural Science, v.119, n.3, p.653-657, 1994.

Iaffe, A.; Pinto, H.; Arruda, F. B.; Quaglia, L.; Sakai, E.; Pires, R. C. M.; Assad, E. Estimativa de temperatura-base e graus-dia com correção pelo fotoperíodo do florescimento à colheita de café em Campinas, SP. In: Simpósio de Pesquisa dos Cafés do Brasil, 2, 2001. Anais... Brasília: Embrapa café, 2001. p.47-47.

Oliveira, P. M. de. Estimativa da evapotranspiração e do coeficiente de cultura do cafeeiro (Coffea arábica L.). Lavras: UFLA, 2003. 86p. Dissertação Mestrado

Ometto, J. C. Bioclimatologia vegetal. São Paulo: Agronômica Ceres 1981. 440p.

Snedecor, G. W.; Cochran, W. G. Statistical methods. 8.ed. Ames: Iowa State University Press, 1989. 503p.

Snyder, R. L. Hand calculating degree days. Agriculture and Forest Meteorology, v.35, n.1/4, p.353-358, 1985.

Wright, J. L. New evapotranspiration crop coefficients. Journal of the Irrigation and Drainage, v.108, n.1, p.57-75, 1982.

Yang, S.; Logan, J.; Coffey, D. Mathematical formulae for calculating the base temperature for growing degree-days. Agricultural and Forest Meteorology, v.74, n.1/2, p.61-74, 1995. 